\title{
ON A FACTORIZATION PROBLEM \\ FOR CONVERGENT SEQUENCES AND ON HANKEL FORMS IN BOUNDED SEQUENCES
}

\author{
P. P. B. EGGERMONT AND Y. J. LEUNG
}

\begin{abstract}
We solve in the negative the following factorization problem of S. Mazur: Can every convergent sequence be written as $z(n)=(n+1)^{-1} \sum_{i=0}^{n} x(i) y(n-i)$, $n=0,1, \ldots$, with convergent sequences $x$ and $y$ ? This problem also yields the solution of another problem of S. Mazur regarding bounded Hankel forms on the space of all bounded sequences.
\end{abstract}

1. Introduction. We consider the following two problems of S. Mazur (Scottish book, Problems 8 and 88; see [4]).

I. Can every convergent sequence $z$ be written as $z=x \square y$, with convergent sequences $x$ and $y$, where

$$
x \square y(n)=(n+1)^{-1} \sum_{i=0}^{n} x(i) y(n-i), \quad n=0,1, \ldots ?
$$

II. Suppose the sequence $b$ is such that

$$
\sum_{i=0}^{\infty} x(i) \sum_{n=i}^{\infty}(n+i+1)^{-1} b(n+i) y(n)
$$

is convergent for all bounded sequences $x$ and $y$. Is then $\sum_{n=0}^{\infty}|b(n)|<\infty$ ?

Problem $\mathrm{I}$ is concerned with the converse of the theorem "If $x$ and $y$ are convergent, then $x \square y$ is convergent", and Problem II is an attempt at characterizing Hankel forms on the space of all bounded sequences. The study of Hankel forms on Hilbert space is a more standard subject, see Bonsall [1] and references therein.

It is quite natural to consider alongside Problem $I$ the following weakened version:

III. Can every convergent sequence $z$ be written as

$$
z=\sum_{k=0}^{\infty} \lambda(k) x_{k} \square y_{k}
$$

with convergent sequences $x_{k}, y_{k}$, and

$$
\sum_{k=0}^{\infty}|\lambda(k)|\left\|x_{k}\right\|\left\|y_{k}\right\|<\infty ?
$$

Received by the editors August 12, 1984 and, in revised form, February 20, 1985.

1980 Mathematics Subject Classification. Primary 40A05, 30B30, 47B35. 
Now it turns out that the Problems II and III are equivalent: Both problems have affirmative answers or both have negative ones. Since II has a negative answer as shown by Kwapien and Pelczyński, Problem III has a negative answer as well, and consequently so does Problem I. Here we provide an alternative simple approach to III and I. The idea is to study the growth of the means.

$$
A(z ; r)=\int_{0}^{2 \pi}\left|\frac{d}{d t} e^{i t \hat{z}}\left(r e^{i t}\right)\right| d t
$$

as $r \rightarrow 1^{-}$, where for every bounded sequence $z$,

$$
\hat{z}(\zeta)=\sum_{n=0}^{\infty} z(n) \xi^{n}, \quad|\zeta|<1
$$

We get different rates of growth for general $z \in c$ or $z \in c \square c$.

The close relationship between II and III comes about by considering bounded linear functionals of the form $\beta(z)=\sum_{n=0}^{\infty} b(n) z(n)$ on $c \square c$. If the interchange in the order of summation is allowed, then $\beta(x \square y)$ is equal to the Hankel bilinear form (1.2), and this would go a long way in proving the equivalence. It turns out that the interchange works, provided $x$ or $y$ are convergent sequences.

In the next section we establish some notations and prove some results about $c \square c$, viz. that $c \square c$ is dense in $c$, and that $\operatorname{span}(c \square c)$ is a Banach space. In $\S 3$ we give our solution to Problems III and I based on the $A(z ; r)$. In $\$ 4$ we show the interchangeability result, from which the equivalence of the two problems follows.

2. Preliminaries. As usual $l^{\infty}, c$ and $c_{0}$ denote the Banach spaces of all sequences which are resp. bounded, convergent, and convergent to zero, with norm $\|x\|=$ $\sup \{|x(i)|: i=0,1, \ldots\}$, and $l^{1}$ is the space of absolutely summable sequences.

We denote the element of $c$ all of whose components equal 1 by 1 . Note that $1 \square 1=1$. For $x \in c$, we let $x_{\infty}=\lim _{i \rightarrow \infty} x(i)$. Observe that for $x, y \in c$, we have $(x \square y)_{\infty}=x_{\infty} y_{\infty}$.

The following inclusions provide standard exercises:

$$
l^{\infty} \square l^{\infty} \subset l^{\infty}, \quad c \square c \subset c, \quad c_{0} \square l^{\infty} \subset c_{0} .
$$

Problem I asks whether $c \square c=c$, and we may wonder about the other inclusions as well. (It will turn out they are all strict.) A first (misleading) step towards an answer is

LEMMA 2.1. $c \square c$ is dense in $c$.

Proof. Let $z \in c$. Then $z=z_{\infty} \cdot 1+y$, with $y \in c_{0}$. For $0<r<1$, let $y_{r}$ be defined by $y_{r}(n)=r^{n} y(n)$. Then $\left\|y-y_{r}\right\| \rightarrow 0$ as $r \rightarrow 1^{-}$, and $y_{r} \in l^{1}$. But then $x_{r}$, defined by $\hat{x}_{r}\left(e^{i t}\right)=e^{-i t}\left\{(d / d t) e^{i t} \hat{y}_{r}\left(e^{i t}\right)\right\}$, is in $l^{1}$ as well. Now $y_{r}=x_{r} \square 1$, and so $z_{r}$ defined by $z_{r}=z_{\infty} \cdot \mathbf{1}+y_{r}$ satifies $z_{r}=\left(z_{\infty} \cdot \mathbf{1}+x_{r}\right) \square \mathbf{1} \in c \square c$, and $\left\|z_{r}-z\right\|$ $\rightarrow 0$ as $r \rightarrow 1^{-}$. So $c \square c$ is dense in $c$.

Later on we also need some properties of $\operatorname{span}(c \square c)$, defined here as

$$
\left\{\sum_{n=0}^{\infty} \lambda(n) x_{n} \square y_{n}\left|\sum_{n=0}^{\infty}\right| \lambda(n) \mid\left\|x_{n}\right\|\left\|y_{n}\right\|<\infty\right\},
$$


$x_{n}, y_{n}$ convergent sequences. For $z \in \operatorname{span}(c \square c)$ define

$$
\|z\|_{\square}=\inf \left\{\sum_{n=0}^{\infty}|\lambda(n)|\left\|x_{n}\right\|\left\|y_{n}\right\| \mid z=\sum_{n=0}^{\infty} \lambda(n)\left(x_{n} \square y_{n}\right)\right\} .
$$

LEMMA 2.2. $\left(\operatorname{span}(c \square c),\|\cdot\|_{\square}\right)$ is a Banach space.

Proof. If $\|\cdot\|_{\square}$ is a norm, it is not hard to see that $\operatorname{span}(c \square c)$ is closed in the $\|\cdot\|_{\square}$ norm. We verify the triangle inequality. Let $a=\sum_{n=0}^{\infty} \lambda(n)\left(x_{n} \square y_{n}\right), b=$ $\sum_{n=0}^{\infty} \mu(n)\left(s_{n} \square t_{n}\right), d=\sum_{n=0}^{\infty} \nu(n)\left(u_{n} \square v_{n}\right)$ be (nearly) “infimal" representations, and $a=b+d$. Then $\sum_{n=0}^{\infty}\left\{\mu(n)\left(s_{n} \square t_{n}\right)+\nu(n)\left(u_{n} \square v_{n}\right)\right\}$ is another representation of $a$, but not necessarily (nearly) infimal, so

$$
\sum_{n=0}^{\infty}|\lambda(n)|\left\|x_{n}\right\|\left\|y_{n}\right\| \leqslant \sum_{n=0}^{\infty}\left\{|\mu(n)|\left\|s_{n}\right\|\left\|t_{n}\right\|+|\nu(n)|\left\|u_{n}\right\|\left\|v_{n}\right\|\right\}
$$

but this says that $\|a\|_{\square} \leqslant\|b\|_{\square}+\|d\|_{\square}$.

3. Estimates for $A(z ; r)$. We prove estimates for $A(z ; r)$ when $z$ is an arbitrary bounded sequence, and when $z \in c \square c$. We then prove that the estimates are sharp. (All integrals are over the interval $[0,2 \pi]$.)

THEOREM 3.1. (i) For $z \in l^{\infty}, 0<r<1, A(z ; r) \leqslant 4 \pi\|z\|\left(1-r^{2}\right)^{-3 / 2}$;

(ii) for $z \in c, \lim _{r \rightarrow 1^{-}} A(z ; r)\left(1-r^{2}\right)^{3 / 2}=0$;

(iii) for $x, y \in l^{\infty}, 0<r<1, A(x \square y ; r) \leqslant 2 \pi\|x\|\|y\|\left(1-r^{2}\right)^{-1}$.

Proof. (i) By the Cauchy-Schwarz inequality

$$
\begin{aligned}
A(z ; r) & \leqslant(2 \pi)^{1 / 2}\left(\int \mid \frac{d}{d t} e^{\left.i t \hat{z}\left(r e^{i t}\right) \mid d t\right)^{1 / 2}}\right. \\
& \leqslant(2 \pi)^{1 / 2}\left(\sum_{n=0}^{\infty}(n+1)^{2}|z(n)|^{2} r^{2 n}\right)^{1 / 2} \\
& \leqslant 4 \pi\|z\|\left(1-r^{2}\right)^{-3 / 2} .
\end{aligned}
$$

(In the second step we used Parseval's relation.)

(ii) Write $z=z_{\infty} \cdot 1+y$, with $y \in c_{0}$. Similar to (i), one proves that $A(y ; r)=$ $o\left(\left(1-r^{2}\right)^{-3 / 2}\right)$, and $A(1 ; r)=2 \pi\left(1-r^{2}\right)^{-1}$. Finally, $A(z ; r) \leqslant\left|z_{\infty}\right| A(1 ; r)+$ $A(y ; r)$ gives estimate (ii).

(iii) Since $(d / d t)\left\{e^{i t}(x \square y) \hat{(}\left(r e^{i t}\right)\right\}=e^{i t} \hat{x}\left(r e^{i t}\right) \hat{y}\left(r e^{i t}\right)$, the Cauchy-Schwarz inequality gives

Now

$$
A(x \square y ; r) \leqslant\left(\int\left|\hat{x}\left(r e^{i t}\right)\right|^{2} d t\right)^{1 / 2}\left(\int\left|\hat{y}\left(r e^{i t}\right)\right|^{2}\right)^{1 / 2} .
$$

$$
\int\left|\hat{x}\left(r e^{i t}\right)\right|^{2} d t=2 \pi \sum_{n=0}^{\infty}|x(n)|^{2} r^{2 n} \leqslant 2 \pi\|x\|^{2}\left(1-r^{2}\right)^{-1},
$$

and a similar estimate for the $\hat{y}$ integral gives the required bound.

It is now apparent that the inclusions in (2.2) are strict if the inequalities in Theorem 3.1(i)-(ii) are sharp. 
THEOREM 3.2. (i) There exists a constant $K>0$ and $z^{*} \in l^{\infty}$ such that $A\left(z^{*} ; r\right) \geqslant$ $K\left(1-r^{2}\right)^{-3 / 2}$ for all $0<r<1$.

(ii) For every decreasing, continuous function $a(r)$ with $a(1)=0$, there exists $z^{*} \in c_{0}$ and a constant $K>0$ such that $A\left(z^{*} ; r\right) \geqslant K\left(1-r^{2}\right)^{-3 / 2} a(r)$ for all $0<r$ $<1$.

Proof. For every $z \in l^{\infty}$, Dunford and Schwartz [2, II. 3.15, IV. 8.5],

$$
A(z ; r)=\sup \mid \int \overline{\hat{b}\left(e^{i t}\right)} e^{-i t} \frac{d}{d t}\left\{e^{\left.i t \hat{z}\left(r e^{i t}\right)\right\} d t \mid}\right.
$$

where the supremum is over all $\hat{b} \in L^{\infty}(0,2 \pi)$, with $\|\hat{b}\|_{L^{\infty}(0,2 \pi)} \leqslant 1$. Writing $\hat{b}\left(e^{i t}\right)$ $=\sum_{n=-\infty}^{\infty} b(n) e^{i n t}$ we get by Parseval's relation

$$
A(z ; r)=\sup 2 \pi\left|\sum_{n=0}^{\infty}(n+1) \overline{b(n)} z(n) r^{n}\right|
$$

Let $c$ be a positive constant, and consider the series

$$
\hat{b}_{N}\left(e^{i t}\right)=\sum_{n=-N}^{N} e^{i c n \log n} e^{i n t}
$$

which satisfies $\left\|\hat{b}_{N}\right\|_{L^{\infty}(0,2 \pi)} \leqslant C N^{1 / 2}$ for all $N$, and for some constant $C$, Zygmund [5, Chapter V, Theorem 4.7]. Let $z^{*} \in l^{\infty}$ be defined by $z^{*}(n)=e^{i c n \log n}$. Then

$$
\begin{aligned}
A\left(z^{*} ; r\right) & \geqslant 2 \pi\left|\sum_{n=0}^{N}(n+1) \overline{b_{N}(n)} z^{*}(n) r^{n}\right| /\left\|\hat{b}_{N}\right\|_{L^{\infty}} \\
& \geqslant 2 \pi C^{-1} N^{-1 / 2} \sum_{n=0}^{N}(n+1) r^{n}
\end{aligned}
$$

for all $r$ and $N$. Taking $N$ to be the integer part of $(1-r)^{-1}$ we get $A\left(z^{*} ; r\right) \geqslant$ $K\left(1-r^{2}\right)^{-3 / 2}$, with $K>0$.

To prove (ii), let $z_{r}$ be defined by $z_{r}(n)=e^{i c n \log n} r^{n}$, then, as before,

$$
A\left(z_{r} ; r\right) \geqslant 2 \pi C^{-1} N^{-1 / 2} \sum_{n=0}^{N}(n+1) r^{2 n} \geqslant K_{1}\left(1-r^{2}\right)^{-3 / 2} .
$$

Consequently, $\left(1-r^{2}\right)^{3 / 2} A\left(z_{r} ; r\right) / a(r) \rightarrow \infty$, for $r \rightarrow 1^{-}$. By the uniform boundedness principle, there exists $z \in c_{0}$ such that $\left(1-r^{2}\right)^{3 / 2} A(z ; r) / a(r) \rightarrow \infty$ as $r \rightarrow 1^{-}$.

Corollary 3.5. $\operatorname{span}(c \square c)$ is not closed in $c$.

COROllary 3.6. All inclusions in (2.1) are strict.

4. The dual of $\operatorname{span}(c \square c)$. In this section we show the equivalence of Problems II and III. Our solution of Problem III then also provides the negative answer to Problem II. The all and only important step is the identification of the dual of $\operatorname{span}(c \square c)$ with all bounded Hankel forms on $c \times c$. 
THEOREM 4.1. A linear functional $\beta$ on $\operatorname{span}(c \square c)$ is bounded iff there exists a $b \in l^{\infty}$ such that

$$
\beta(z)=\sum_{n=0}^{\infty} b(n) z(n), \quad \forall z \in \operatorname{span}(c \square c),
$$

and the Hankel form

$$
B(x, y)=\sum_{i=0}^{\infty} x(i) \sum_{n=0}^{\infty}(n+i+1)^{-1} b(n+i) y(n)
$$

is bounded on $c \times c$.

Proof. Only if. Let $\beta$ be a bounded linear functional on $\operatorname{span}(c \square c)$; then it is also bounded on $\operatorname{span}\left(c_{0} \square c_{0}\right)$. Let $x, y \in c_{0}$, and define $x_{m} \in c_{0}$ by $x_{m}(i)=x(i)$ for $i \leqslant m$, and zero otherwise. Then

$$
\beta(x \square y)=\lim _{m, k} \beta\left(x_{m} \square y_{k}\right)=\lim _{m, k} \sum_{n=0}^{\infty} b(n)\left(x_{m} \square y_{k}\right)(n)
$$

for some $b \in l^{\infty}$. Note that the infinite sum is in fact a finite sum. So, $\beta(z)$ is given by (4.1) when $z \in \operatorname{span}\left(c_{0} \square c_{0}\right)$. Interchanging the order of summation yields

$$
\beta\left(x_{m} \square y_{k}\right)=\sum_{i=0}^{m} x(i) \sum_{n=0}^{k}(n+i+1)^{-1} b(n+i) y(n) .
$$

We denote the right-hand side by $B_{m, k}(x, y)$. From the boundedness of $\beta$ the uniform boundedness of the $B_{m, k}$ follows, and thus upon the successive evaluations of the limits in $B(x, y)=\lim _{m, k \rightarrow \infty} B_{m, k}(x, y)$, we have that

$$
B(x, y)=\sum_{i=0}^{\infty} x(i) \sum_{n=0}^{\infty} b(n+i)(n+i+1)^{-1} y(n)
$$

is well defined, and is a bounded bilinear form on $c_{0} \times c_{0}$. It is now a standard exercise to show that the recipes (4.1-4.2) for $\beta$ and $B$ apply also on $\operatorname{span}(c \square c)$ and $c \times c$, and that $B$ is a bounded bilinear form on $c \times c$.

The If part is substantially similar to the above, and is left to the reader.

\section{COROllary 4.2. Problems II and III are equivalent.}

Proof. The only complication is whether a bounded Hankel form on $c \times c$ is also bounded on $l^{\infty} \times l^{\infty}$ (and is given by the "same" recipe), but this is quite straightforward. Otherwise, the corollary is a consequence of the fact that the dual of $c$ (or a closed subspace thereof) is $l^{1}$ (or a quotient space of $l^{1}$, Dunford and Schwartz [2, II, 3.11]). Note that if $\operatorname{span}(c \square c)$ is a closed subspace of $c$, it equals $c$, since it is dense in $c$, see Lemma 2.1 .

5. Final remarks. With regard to Problem I, a good partial success would be to establish whether $c \square c$ is a linear space or not. If $c \square c$ is to equal $c$, it had better be one. The necessary conditions of Lemma 3.1(iii) cannot distinguish $c \square c$ from $\operatorname{span}(c \square c)$, so they are useless in settling this question. Another question is as follows: We have shown that $x \square X \neq X$ for $X=c$ and $l^{\infty}$. Are there interesting sequence spaces $X$ for which there is equality, or for which $X \square X$ is a linear space?

ACKNOWLEDGMENT. The first author was partially supported by United States Army under Contract No. DAAG 29-83-K-0109. 


\section{REFERENCES}

1. F. F. Bonsall, Boundedness of Hankel matrices, J. London Math. Soc. (2) 29 (1984), 289-300.

2. N. Dunford and J. T. Schwartz, Linear operators. Part I, Interscience, New York, 1957.

3. S. Kwapien and A. Pelczyński, The main triangle projection in matrix spaces and its applications, Studia Math. 34 (1970), 43-68.

4. R. D. Mauldin (ed.), The Scottish book, Birkhäuser, Boston, Mass., 1979.

5. A. Zygmund, Trigonometric series (Second Ed.), Vol. I, Cambridge Univ. Press, Cambridge, 1959.

Department of Mathematical Sciences, University of Delaware, Newark, Delaware 19716 